\section{IN THE NEWS}

This year's Nobel prize as reported in La Stampa.

The Nobel on Medicine to three explorers of the brain

[Eric] Kandel's research on memory is fascinating. In the 1950s, physiologists were still arguing over the seat of our memories ... In 1953, a 27-year old patient suffering from epilepsy, identified by the initials H. M., underwent removal of a part of his brain that included almost all the hippocampus. As a result, ... H. M. lost the capacity to fix new memories - his life had stopped at 27 years. Could the hippocampus be the seat of memory? What happens when we fix a memory? Kandel a 70-year old professor at Columbia University thought that this very complex problem could be addressed by using a simple biological model. He found this [model] in a sea slug, Aplysia californica, a mollusc with twenty thousand brain cells, five million times fewer than us. Kandel discovered that when Aplysia registers an experience, some synapses, that is, contacts between neurons are stabilized.

Piergiorgio Strata, a neuroscientist at the University of Turin, remembers one of the first times Kandel presented his results, in 1967. "At the talk - Strata says - "was also John Eccles, who had already received the Nobel Prize in 1963 ... Eccles was very open to the work of his younger and more creative colleagues. He was excited by Kandel's work and said to me: 'Kandel's approach to the problem will win him a Nobel prize.' Eccles died three years ago and will never know. Still, he had foreseen it, $\mathbf{3 3}$ years earlier."

Translated by Tanita Casci, Nature Reviews Genetic

\title{
Arrowing into the heart of development
}

Wnt proteins are a class of secreted signalling molecules that have an extensive function in patterning and morphogenesis during invertebrate and vertebrate development. Important progress has been made in the identification of the cellular pathways that mediate the various effects of the Wnt family. Now, observations made on three species provide compelling evidence that low-density lipoprotein (LDL)-receptor-related proteins (LRP) are also involved in the transmission of Wnt signals. Furthermore, the involvement of another LDLreceptor family member in development raises the possibility that this class of proteins has a general function during embryogenesis.

First, Wehrli et al. studied a Drosophila melanogaster mutant called arrow with a phenotype identical to the one observed in flies lacking Wingless $(\mathrm{Wg}$ ), a canonical member of the Wnt family. The authors found that Arrow is a protein homologous to mammalian LRP5 and LRP6. Arrow was found to be required in the cells receiving the $\mathrm{Wg}$ protein and to act upstream of Dishevelled, a well-known Wg transducer, indicating that it could function as a $\mathrm{Wg}$ receptor. Indeed, overexpression of either the Wg receptor Drosophila frizzled $2(d f z 2)$, or arrow, has equivalent effects in the Drosophila wing. In addition, overexpression of $d f z 2$ alone cannot rescue the arrow mutation, further indicating that both molecules act as Wg co-receptors. Tamai et al. extended these observations to vertebrates by studying Xenopus embryos, where Wnt signalling is known to participate in dorsal axis and neural crest formation. The ventral injection of LRP6 messenger RNA to frog embryos induced a secondary dorsal axis (top panel: control (a) and injected (b)), expansion of the neural crest and expression of several Wntresponsive genes. Similarly, the expression of a dominant-negative form of LRP6 inhibited crest development. As in the case of Arrow in Drosophila, LRP6 also acted upstream of Dishevelled in frog embryos. However, Tamai et al. obtained more direct evidence that LRP6 functions as a Wnt co-receptor, by showing that an antibody against $\mathrm{mFz8}$, a Xenopus Wnt receptor, can precipitate LRP6 only in the presence of Wnt-1, indicating that these two proteins and $\mathrm{mFz8}$ can form a macromolecular complex.

The involvement of LRP6 in Wnt signalling is not restricted to lower vertebrates but can also be observed in mammals where, more importantly, LRP6 seems to mediate the action of several Wnt proteins. Pinson et al. observed a marked similarity between the phenotype of LRP6 mutant mice and defects reported for various Wnt mutations. LRP6 mutants lacked a tail (as do Wnt-3a mutants), showed limb malformations (as do Wnt-7a mutants) and, similar to Wnt-1 mutants, had midbrain/hindbrain defects


Top panel: Control frog embryo at stage-40 (a). Ventral injection of $\angle R P 6$ mRNA induced trunk duplication lacking head and the notocord (b). Figure adapted fromTamai, K. et al. Bottom panel: Mouse embryos. The isthmus (arrow) demarcating the mid/hindbrain of wild-type embryos (c) is less distinct in mutants (d). Figure adapted from Wehrli, M. et al. (c) (2000) Macmillan Magazines Ltd.

(bottom panel: wildtype (c) and LRP6 mutant (d)). In addition, the tail phenotype was exacerbated in Wnt-3a/LRP6 double mutants, suggesting once again that both molecules act in the same signalling pathway.

The LDL receptor family has long been known to function in the internalization of lipoproteins. However, a large body of evidence has already shown that the different members of the family are also involved in brain development. In particular, the very low-density lipoprotein receptor and the Apolipoprotein E2 receptor have been identified as components of the reelin signalling pathway, as their absence leads to cerebellar abnormalities akin to those observed in reeler mice. The discovery of a role for LRPs during embryogenesis extends the list of LDL-receptor family members involved in development. This finding should prompt us to revisit the embryological defects known to occur in knockouts of other members of the family, such as the holoprosencephaly observed in megalin-deficient mice. Undoubtedly, the above results provide clear evidence that on this occasion the arrow has hit the mark.

Juan Carlos López

(2) References and links ORIGINAL RESEARCH PAPER Wehrli, M. et al. arrow encodes an LDLreceptor-related protein essential for Wingless signalling. Nature $\mathbf{4 0 7}$, 527-530 (2000) | Tamai, K. et al. LDL-receptor-related proteins in Wnt signal transduction. Nature 407, 530-535 (2000) | Pinson, K. I. et al. An LDLreceptor-related protein mediates Wnt signalling in mice. Nature $\mathbf{4 0 7}$ $527-530$ (2000)

FURTHER READING Wodarz, A. \& Nusse, R. Mechanisms of Wnt signalling in development. Annu. Rev. Cell Dev. Biol. 14, 59-89 (1998) | Herz, J. \& Beffert, U. Apolipoprotein E receptors: linking brain development and Alzheimer's disease. Nature Rev. Neurosci. 1, 51-58 (2000) 